\title{
Ensuring the Consistency of Competitive Strategy and Logistic Performance Management
}

\author{
Gregor von Cieminski and Peter Nyhuis \\ Institute of Production Systems and Logistics (IFA), \\ Gottfried Wilhelm Leibniz Universität Hannover, \\ An der Universität 2, D-30823 Garbsen, Germany \\ Email: \{cieminski,nyhuis\}@ifa.uni-hannover.de
}

\begin{abstract}
Manufacturing companies often merely attain reduced levels of logistic performance due to inconsistencies between their logistic objectives, logistic performance targets and logistic performance management actions. Qualitative influence models represent the inter-dependencies between these factors on two levels: firstly between logistic objectives, secondly between logistic performance measures and logistic planning and control parameters. Expert knowledge gained from modelling the dynamic behaviour of logistic systems facilitates the interpretation and evaluation of the qualitative influence models. On this basis, consistent logistic objectives can be defined and objectiveoriented planning and control actions can be identified. The modelling insights are integrated into a logistic performance diagnosis procedure and tool that improve logisticians' understanding of the consequences of their management decisions and enhance the closed-loop management of logistic performance.
\end{abstract}

Keywords

Logistic performance, performance management, qualitative model

\section{Introduction}

Manufacturing companies compete with each other in terms of product functionality, product and service flexibility, price, product quality and logistic performance [1]. Competitive advantages in terms of the first two factors originate from the superior strategic design of products or production systems, respectively. Advantages in terms of the latter three factors firstly require the setting of adequate strategic objectives. Secondly, they also depend on superior operational performance. In order to attain the performance levels required, manufacturing companies have to ensure that their 
competitive strategies and their operational performance management actions are consistent [2]. This especially applies to the context of logistic performance: Wiendahl et al. [3] name inconsistencies between logistic performance objectives and operational planning and control parameters as one of the root causes of logistic performance deficits. As logistic performance has become an important customer purchase criterion [4] and developed into a proven success factor for manufacturing companies [5], the companies have to be equipped with performance management approaches that support them in avoiding these inconsistencies.

As a solution, this paper proposes qualitative logistic influence models and an approach for logistic performance diagnosis. It first provides a general overview of the consequences that strategic logistics management decisions have for operational logistic performance management. Second, it introduces two types of qualitative influence models that represent the inter-dependencies between logistic objectives as well as between logistic performance measures and logistic planning and control parameters. General insights gained from the interpretation and evaluation of these models are summarised. Third, as practical applications of the influence models, the paper presents a diagnostic procedure and software tool for factors affecting the operational logistic performance of manufacturing companies.

\section{Linking Strategic and Operational Logistics Management}

Given the market developments described in section 1, many manufacturing companies adopt competitive strategies based on their logistic performance; their logistic strategies become competitive strategies. Strategic logistics management has two main tasks: the formulation of strategic logistic objectives and the design of logistic and production systems. Both of these tasks have repercussions for operational logistics management. The primary logistic objectives of a manufacturing company depend on its product-delivery strategy, i.e. whether it satisfies customer demands from a finished-products store or whether it fulfils specific customer orders directly from production. Accordingly, the company's operational performance objectives and the ensuing planning and control actions have to be derived from the primary objectives "high service level" and "low delivery delay" or "high delivery reliability" and "short lead times", respectively.

The product-delivery strategy also influences a design aspect of manufacturing companies' logistic systems: the inclusion or omission of the distribution-logistics process. In the more detailed design of the three logistic processes of manufacturing companies - procurement, production and distribution logistics - it is standard practice to follow the prescriptions of logistic reference models. These models typically provide a choice of several process models as design templates for each of the logistic processes. An analysis of the process reference models developed by the Siemens concern [6] on the basis of the supply-chain operations reference model [7] led to the derivation of so-called logistic configurations of manufacturing companies [8] (see fig. la and $1 \mathrm{~b}$ ). These configurations indicate those logistic processes that play an active part in the fulfilment of the logistics function. Obviously, only logistically-active processes pursue explicit logistic objectives, carry out logistic 
planning and control actions, and therefore have an impact on the logistic performance of a manufacturing company. Hence, the specific logistic configuration of a company shapes the basic structure of the relevant logistic inter-dependencies (see fig. 1d). In turn, this structure determines the exact operational performance objectives and planning and control actions whose consistency has to be achieved.

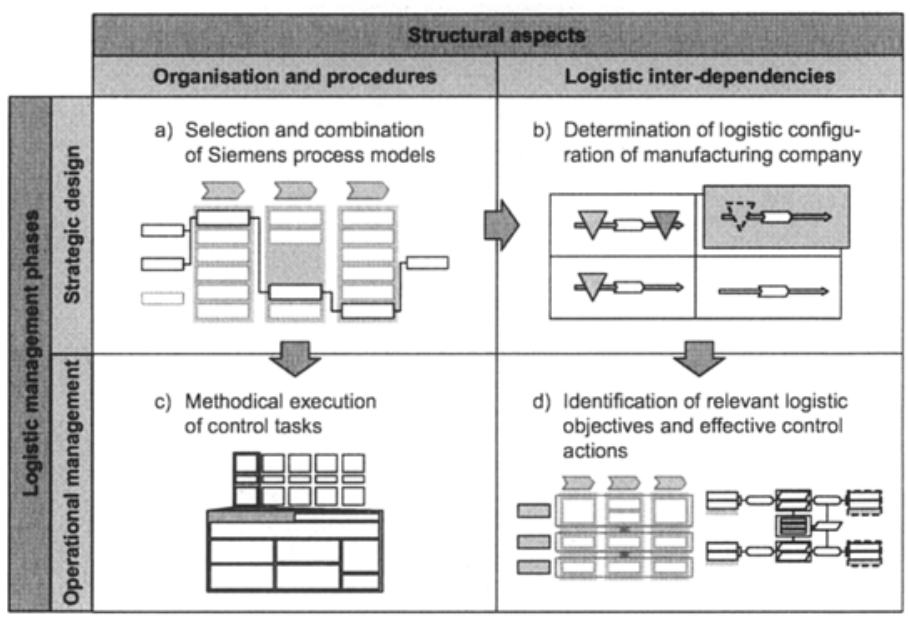

Fig. 1. Configurations of industrial-enterprise logistics determine the basic structure of the logistic inter-dependencies

To ensure the general applicability of the analysis, this paper considers a "maketo-stock" configuration, in which all processes affect the logistic performance.

\section{Modelling and Analysing Logistic Inter-dependencies}

Generally, analytical models of logistic processes are used to describe and analyse logistic inter-dependencies. These models often fall short of clearly establishing the relationships between logistic performance measures and the operational planning and control actions that the logistic processes can take. Moreover, inter-dependencies between separate logistic processes are not comprehensively taken into account. To overcome these shortcomings, qualitative influence models that holistically identify logistic inter-dependencies within and across process boundaries were compiled [8]. The models are based on the Theory of Logistic Operating Curves developed at the Institute of Production Systems and Logistics (IFA) [9]. This theory includes a range of analytical models of the logistic behaviour of inventories and production systems. Using the mathematical relationships of the models, it is possible to establish logistic inter-dependencies on two levels -between logistic objectives (see section 3.1) and between logistic performance measures and parameters that are manipulated through planning and control actions (see section 3.2). 


\subsection{Inter-dependencies between Logistic Performance Objectives}

IFA has developed sets of logistic objectives for both production and storage processes. For isolated logistic processes, a qualitative characterisation of the interdependencies between the objectives is available [9]. As an extension, fig. 2 shows the combination of the sets of objectives of the three logistic processes of manufacturing companies. As for the separate processes, the logistic objectives can be assigned to the two directions logistic performance and logistic costs. The latter can further be differentiated into process costs and inventory costs.

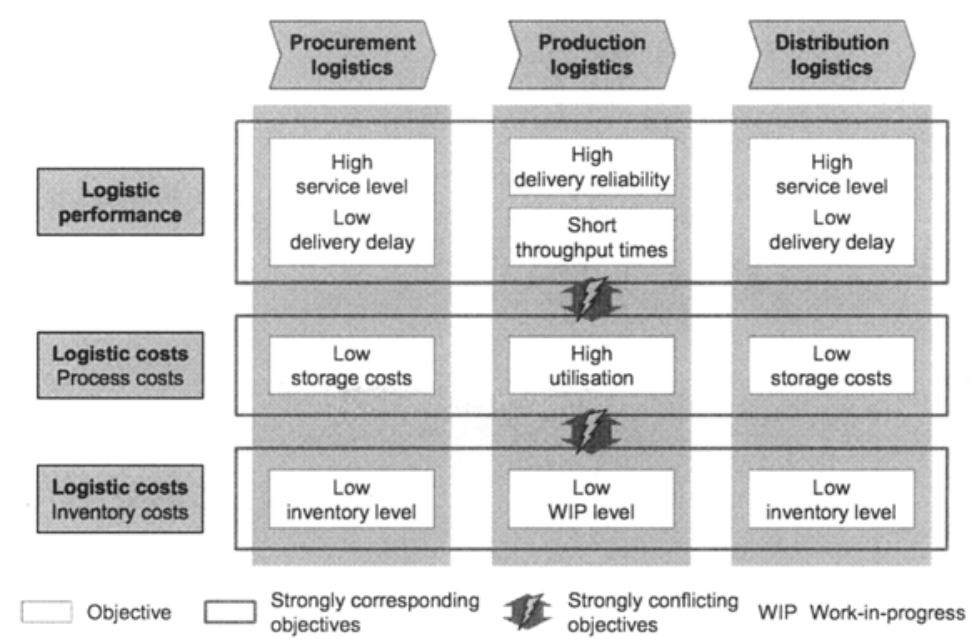

Fig. 2. Overview of inter-dependencies between logistic objectives

The figure also summarises the results of a first qualitative analysis of the interdependencies carried out by means of deductive reasoning. The main insight was that the objectives of each of the three directions correspond to each other across all three logistic processes. Conversely, marked conflicts were found to exist between the objectives of the directions logistic performance and process costs as well as process costs and inventory costs. Accordingly, fig. 2 categorises the inter-dependencies between the objectives into strong correspondences and strong conflicts. (More detailed results of the analysis are provided in [8].)

\subsection{Inter-dependencies between Planning and Control Actions and Logistic Performance Measures}

The effects of operational planning and control actions on the logistic performance of manufacturing companies are modelled through an analogy between technical control processes and logistic performance management. Accordingly, logistic interdependencies are illustrated in qualitative influence models that are comparable to 
the block diagrams known from control-systems theory. An example is shown in fig. 3, the influence model of the production-logistics process adapted from [10].

The qualitative influence models depict the cause-and-effect chains linking the logistic performance measures to the parameters modified by logistic planning and control actions, the so-called determinants. Referring to an example from fig. 3, the performance measures of production-logistics processes "throughput time" and "utilisation" are modelled as functions of the controlled variable "WIP level". The latter is calculated as the difference between the two manipulated variables "actual input" and "actual output". The values of the manipulated variables are in turn controlled by determinants: the actual input by the determinants "actual work content released" and "actual release date", the actual output by the determinants "actual capacity provided" and "date of capacity provision". Obviously, the first pair of determinants is parameterised as part of the order release, while the second pair depends on capacity-control decisions.

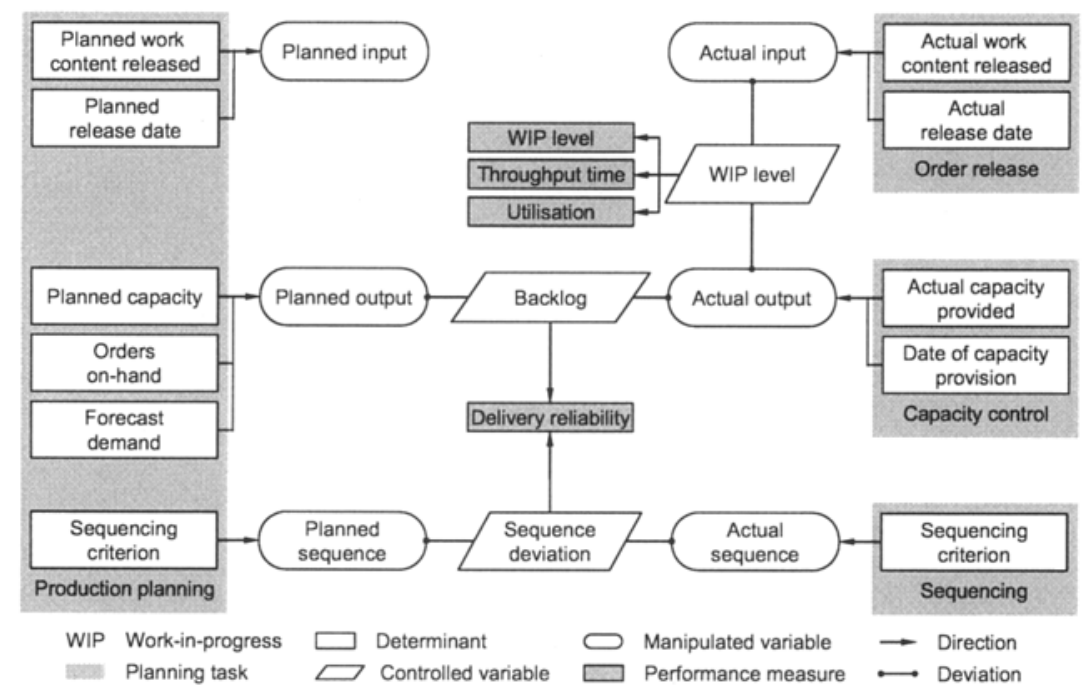

Fig. 3. Qualitative influence model of production planning and control (adapted from [10])

For the inventory management of procurement and distribution logistics processes, a similar influence model has been developed. Additionally, the coupling variables of the logistic processes have been identified so that cause-and-effect chains that extend across process boundaries may be traced as well. Through the use of these models, logisticians can identify all planning and control actions affecting a certain performance measure. Vice versa, they can locate all performance measures that are affected by a certain planning and control action. 


\subsection{Qualitative Evaluation of Logistic Inter-dependencies}

The Theory of Logistic Operating Curves not only determines the structure of the qualitative influence models presented above. IFA's expertise in the interpretation of the quantitative models of the theory also facilitates a qualitative evaluation of the logistic inter-dependencies [8]. This process results in two types of guidelines for logistic performance management: guidelines for the consistent definition of logistic objectives and guidelines for the identification of objective-oriented logistic planning and control actions. Examples for both types are given below:

- The logistic processes of manufacturing companies should jointly pursue corresponding objectives in the directions shown in fig. 2. Conversely, the combined pursuit of objectives from conflicting directions should be avoided.

- Certain target values of logistic processes constitute values of planning parameters in other processes. The value settings for both have to be consistent.

- A logistic process's own planning and control actions are most effective for improving its logistic performance, both in magnitude and responsiveness.

- For maximizing logistic performance, manufacturing companies have to achieve objective-orientation and consistency of planning and control actions across all logistic processes. This is also essential for achieving very good performance levels at low logistic costs.

- Planning and control actions manipulating the magnitude and timing of inputs and outputs of production resources and stores are most effective in improving their logistic performance.

- Sequencing decisions and dedicated capacity adjustments are the most effective planning and control actions from the perspective of single customer orders.

On the premise that the logisticians responsible adhere to these guidelines, manufacturing companies can ensure the application of logistic performance management practices that are both consistent and effective.

\section{Using Qualitative Models for Logistic Performance Diagnosis}

In order for manufacturing companies to be able to directly apply the qualitative influence models and the guidelines for logistic performance management, IFA developed a logistic performance diagnosis procedure and tool on their basis.

A knowledge base containing the insights gained from modelling and analysing the logistic inter-dependencies lies at the core of the diagnostic procedure. On the one hand, this encompasses the underlying structure of the logistic interdependencies. For example, the cause-and-effect chains leading from the logistic performance measure "throughput time" via the corresponding controlled and manipulated variables to determinants such as "actual capacity provided" or "date of capacity provision" are represented (see fig. 3). On the other hand, the knowledge base includes the guidelines for logistic performance measurement and other results of the evaluation of the inter-dependencies. These are represented by logical statements such as: "The actual capacity provided is the second most effective determinant to influence the performance measure delivery reliability." 
In the diagnostic tool, the contents of knowledge base are translated into relational databases. Their development and maintenance is facilitated through the expert interface shown at the bottom of fig. 4. Logistics experts are able to modify the structure of the databases and to enter new information into the database tables.

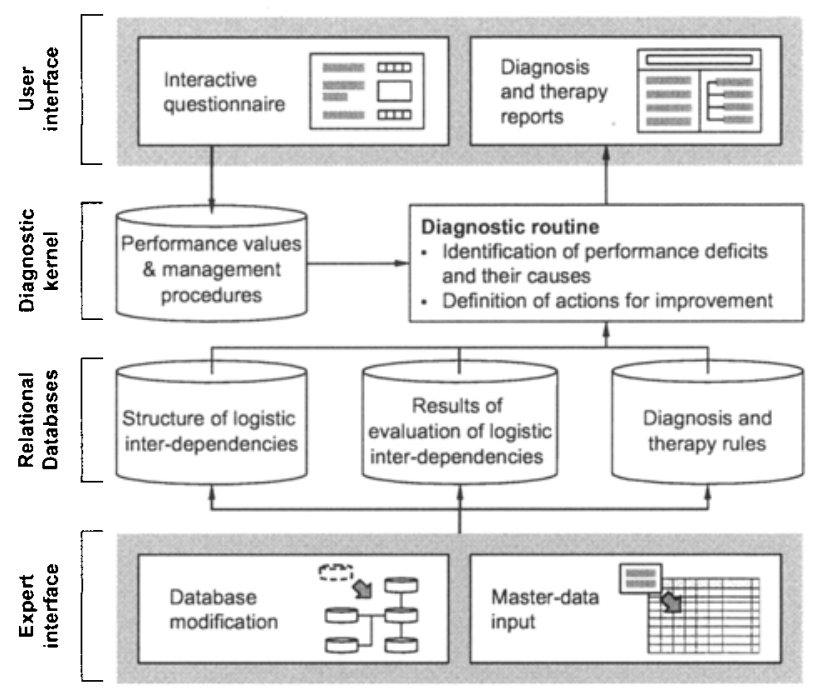

Fig. 4. Structure of the diagnostic software prototype

The application of the diagnostic procedure and tool comprises three phases: performance appraisal, diagnostic analysis and therapy definition. In the first phase, performance data of the manufacturing company under consideration are collected. This is facilitated through the input section of the user interface of the diagnostic tool shown at the top of fig. 4. An interactive questionnaire provides a structure for the required information on the logistic performance. The logistic configuration, target and actual performance values as well as the logistic planning and control practices applied are recorded. At this stage, logistic performance deficits become apparent.

In the second phase, the performance data are analysed using the databases of the diagnostic software tool. A diagnostic routine systematically determines the causes of logistic performance deficits. The routine identifies inconsistencies between logistic objectives as well as inconsistencies between logistic performance measures and logistic planning and control actions. The diagnosis also points out instances, in which the planning and control practices applied do not maximise logistic performance.

In the third phase, therapy rules contained in the database are utilised to ensure the consistency of logistics management practices by defining actions for improvement of the current logistic performance. The causes of logistic performance deficits and the recommendations of actions for improvement are highlighted by reports which can by accessed through the user interface (see top of fig. 4). 


\section{Conclusions}

The qualitative logistic influence models presented in this paper are a practical means to ensure the consistency of logistic performance-based competitive strategies and operational logistic performance management. Most importantly, the models help manufacturing companies identify those operational planning and control actions, with which they can effectively improve specific logistic performance measures. The fact that the models consider inter-dependencies between logistic processes - as well as those within the processes - is an added advantage. Thus, consistent strategic and operational logistics management is facilitated across all logistic processes of a manufacturing company.

Transcending the current state of development, two important enhancements of the qualitative influence models are possible. First, the production processes may be disaggregated into combinations of fabrication and assembly processes with variable numbers of production stages and shifting customer-order decoupling points $[6,11]$. The development and analysis of the qualitative models should be extended to the resulting more detailed logistic configurations of manufacturing companies. Second, the qualitative models may serve as a basis for the development of additional quantitative logistic models. For example, it is feasible to extend the Theory of Logistic Operating Curves by establishing and verifying the mathematical relationships between the operational planning and control parameters and the logistic performance measures.

\section{References}

1. B.H. Maskell, Performance Measurement for World Class Manufacturing: A Model for American Companies (Productivity Press, Cambridge, 1991).

2. J. Mills, K. Platts, A. Neely, H. Richards, and M. Bourne, Strategy and Performance: Creating a Winning Business Formula (Cambridge University Press, Cambridge, 2002).

3. H.-H. Wiendahl, G. von Cieminski and H.-P. Wiendahl, Stumbling blocks of PPC: towards the holistic configuration of PPC systems, Production Planning and Control, 7 (16) 634$651(2005)$.

4. A. Maurer and W.A. Stark, Steering Carmaking into the 21st Century: From Today's Best Practices to the Transformed Plants of 2020 (Boston Consulting Group, Boston, 2001).

5. G. Stalk and T.M. Hout, Competing against Time: How Time-Based Competition Is Reshaping Global Markets ( $2^{\text {nd }}$ edition, Free Press, New York, 2003).

6. P. Nyhuis and C. Wolter, Quantifying the Rationalization Potential in Logistics through Supply Chain Design, in: Performance Measurement for Increased Competitiveness, edited by H.-P. Wiendahl and G. von Cieminski (Leibniz Universität Hannover, Hanover, 2002), pp. 14-23.

7. Supply Chain Council, Supply-chain Operations Reference-model Version 8.0 (May 15, 2007), http:/www, supply-chain.org.

8. G. von Cieminski, Einsatz qualitativer Wirkmodelle zur Lenkung der industriellen Unternehmenslogistik ( $\mathrm{PhD}$ thesis, Leibniz Universität Hannover, Hanover, 2007).

9. P. Nyhuis and H.-P. Wiendahl, Fundamentals of Production Logistics (Springer, Berlin et al., 2007, in press).

10. H. Lödding, Verfahren der Fertigungssteuerung (Springer, Berlin et al., 2005).

11. J. Olhager, Strategic positioning of the order penetration point, International Journal of Production Economics, 3 (85) 319-329 (2003). 